\title{
An Analytical, Temperature-dependent Model for Majority- and Minority-carrier Mobility in Silicon Devices*
}

\author{
SUSANNA REGGIANI, MARINA VALDINOCI, LUIGI COLALONGO, \\ MASSIMO RUDAN ${ }^{\dagger}$ and GIORGIO BACCARANI \\ Dipartimento di Elettronica, Informatica e Sistemistica (DEIS) of the University of Bologna, \\ viale Risorgimento 2, 40136 Bologna, Italy
}

(Received 16 December 1998; In final form 14 December 1999)

\begin{abstract}
A new analytical model for carrier mobility in silicon is presented, which is strongly oriented to CAD and suitable for implementation in device simulators. The effects of the electric field, temperature, and doping concentration are accounted for. In particular, the model unifies the descriptions of majority- and minority-carrier mobility and includes the full temperature dependence. The effects of a high longitudinal field are included in the conventional velocity-saturation form; the doping dependence is also incorporated in the latter. The model has been worked out starting from a preliminary investigation using a Boltzmann solver, and has been validated by a number of comparisons with published experiments on silicon.
\end{abstract}

Keywords: Semiconductor-device simulation, bulk mobility model, majority- and minority-carriers, temperature dependence

\section{INTRODUCTION}

The need of accurate analytical models for the transport coefficients in semiconductors, in particular mobility, is becoming more and more exacting, following the miniaturization trend in the integrated-circuit technology and the parallel broadening of the application fields. Also, the increase in the doping concentration makes the difference between the majority- and minoritycarrier scattering more relevant, making it necessary to distinguish these two quantities in the mobility models. On the other hand, in applications of integrated circuits where large current densities are present or electrostatic-discharge phenomena may occur, it is necessary to account for a substantial increase in the devices' operating temperature. This in turn reflects into a need of

*This work has been partly supported by the European Commission under the ESPRIT-23643 Project (ESDEM).

${ }^{\dagger}$ Corresponding author. Tel.: +390512093016, Fax: +390512093073, e-mail: mrudan@deis.unibo.it 
an accurate modeling of the dependence of mobility on temperature. In this paper a physicallybased, analytical mobility model for silicon is presented, incorporating the distinction between majority and minority carriers, the partialionization and high-field effects, and the dependencies on the lattice temperature up to $800 \mathrm{~K}$.

Previous investigations on bulk mobilities have been carried out using the Boltzmann solver based on the expansion of the distribution function in spherical harmonics (SHE) [1]. By incorporating a full-band structure within the Boltzmann solver and implementing the most important scattering mechanisms which affect carrier mobility (i.e., acoustic- and optical-phonon, and impurity scattering), accurate values of the average carrier drift velocity $v$ for any given values of the electric field $E$, doping concentration $N$, and temperature $T$ are determined in a bulk system. The carrier mobility can thus be easily extracted, consistently with the model, as $\mu(T, N, E)=v / E$. By properly tuning the coupling constants and the phonon energy which appear in the scattering terms, an excellent agreement is obtained with existing experimental data over a wide range of operating conditions [2].

The new physically-based model has been developed starting from the SHE calculations validated by comparisons with experiments. More specifically, the temperature dependence of bulk mobilities for both majority and minority carriers has been carefully developed. As a result, a wider range of operating conditions has been investigated, as illustrated below.

The available experimental data, at least in the authors' knowledge, are limited to a temperature range below $500 \mathrm{~K}$ for majority carriers, while no experiments are available for minority carriers outside room temperature. In a first phase, the parameters of the SHE code have been tuned to reproduce the experimental mobilities. Special care has been devoted to the analysis of the temperature dependence of such parameters. This made it possible to extend the sHE predictions over a wider range (up to $800 \mathrm{~K}$ ) than that in which experiments are available. In the second phase, the analytical mobility model has been worked out up to $800 \mathrm{~K}$. Within this temperature range, the mobility data have been extrapolated by the numerical model and fitted with the analytical one.

As far as the dependence on the doping concentration is concerned, the SHE calculations have been compared with sets of experiments from the literature, related to mobility as well as resistivity against temperature for different doping values. The agreement shown by the numerical results with experiments confirms that the temperature and doping dependencies for bulk mobility are correctly predicted by SHE. The same procedure described above with respect to the temperature dependence has been carried out here to analyze the dependence on the doping concentration. The analytical model for carrier mobility has been compared with SHE data in order to fit the characteristics for majority and minority carriers on a wide range of doping concentrations. In particular, as the impurity concentration increases, the mobility profile has a continuously decreasing behavior that is very well described by the empirical model [3], based on a set of measurements at room temperature. Starting from that model, a first investigation on the mobility profile at very high doping concentrations has been made, whereas the analytical model has been developed from [3] adding a number of temperature dependencies, based on physical considerations.

High electric-field effects have been included into the model in the assumption that the carrier saturation velocity is independent of doping concentration and of the transverse electric field. A fitting procedure has been carried out for both majority and minority carriers starting from the numerical data. Previous investigations on impactionization scattering carried out with SHE showed a good agreement with experiments for both the drift-velocity $v s$. electric field characteristic and the impact-ionization coefficients: data up to $5 \cdot 10^{5}$ $\mathrm{V} / \mathrm{cm}$ have been fitted with the analytical model.

Section 2 illustrates the derivation of the bulkmobility model. Section 3 illustrates the dependence of the majority- and minority-carrier mobility 
on the impurity concentration and temperature. Finally, the high electric-field effects are discussed in Section 4.

\section{BULK MOBILITY MODEL AT LOW ELECTRIC FIELD}

The model for the bulk mobility $\mu_{b}$ has been developed starting from the empirical model of [3] in order to take advantage of the fitting performed with experiments at room temperature on a wide range of doping concentrations. The temperature dependence has then been extended by fitting experimental data. The model reads

$$
\begin{aligned}
\mu_{b}(N, T)= & \mu_{0}(T)+\frac{\mu_{L}(T)-\mu_{0}(T)}{1+\left(N / C_{r}(T)\right)^{\alpha}} \\
& -\frac{\mu_{1}(T)}{1+\left(C_{S}(T) / N\right)^{2}},
\end{aligned}
$$

where $N$ is the doping concentration $\left(N_{A}\right.$ or $\left.N_{D}\right)$, $\mu_{L}(T)$ is the lattice mobility limited by acousticand optical-phonon scattering, while $\mu_{0}(T)$ and $\mu_{1}(T)$ give the minimum values for the carrier mobility at high doping concentrations. As shown in Figures 7 and 8 below, the mobility characteristics have a first "step" at about $10^{20} \mathrm{~cm}^{-3}$, followed by a decreasing tail. The third term in (1) accounts for the decreasing of $\mu_{b}$ at the highest values of $N$. A more detailed description of the contributions to the model is given in the following.

\subsection{Analysis of Intrinsic Electron and Hole Mobility}

At very low impurity concentrations the carrierphonon scattering mechanisms are dominant in the intrinsic mobility. Several sets of experiments up to $500 \mathrm{~K}$ show that the mobility of both species of carriers is well described by a relation of the form $\mu_{L}=\mu_{L 0}(T / 300)^{-\gamma}$, with $2.2<\gamma<2.3$, due to the contribution of both acoustic- and optical-phonon scattering. The temperature-dependence investigation has been extended up to $800 \mathrm{~K}$ using the extrapolated data from numerical simulations by SHE, leading to the following law for both electrons and holes:

$$
\mu_{L}(T)=\mu_{L 0}\left(\frac{T}{300}\right)^{-\gamma+c T / 300}
$$

where $\mu_{L 0}$ is the value of the lattice mobility at room temperature, and $c$ provides a slight correction to the mobility at higher temperatures for electrons only. The behavior of (2) can be observed in Figures 1 and 2, where the intrinsic mobility for electrons and holes as a function of temperature is reported. The values of the parameters $\mu_{L 0}, c$ and $\gamma$ are presented in Table $I$. The relative departure of the mobility laws for electrons and holes in terms of the lattice temperature can be ascribed to the different structure of the conduction and valence bands.

\subsection{The Mobility Dependence on the Doping Concentration}

In the low-field regime, carrier mobility is strongly influenced by the ionized-impurity scattering. The theoretical investigation on low-field mobility, carried out using the deterministic Boltzmann solver SHE, has given meaningful explanations about the carrier behavior at very high doping concentrations and temperatures. Experimental data available in the literature against temperature and impurity concentrations were used to carry out comparisons with the numerical model, which turned out to provide a generally-good agreement within the temperature range between 100 and $500 \mathrm{~K}$. It is worth clarifying that the temperature-dependence discussed here is related to the doping-dependent part of the model, and adds up to that embedded in the lattice mobility discussed above.

In the analysis of the temperature dependence of the ionized-impurity scattering mechanism, the partial ionization has been taken into account 


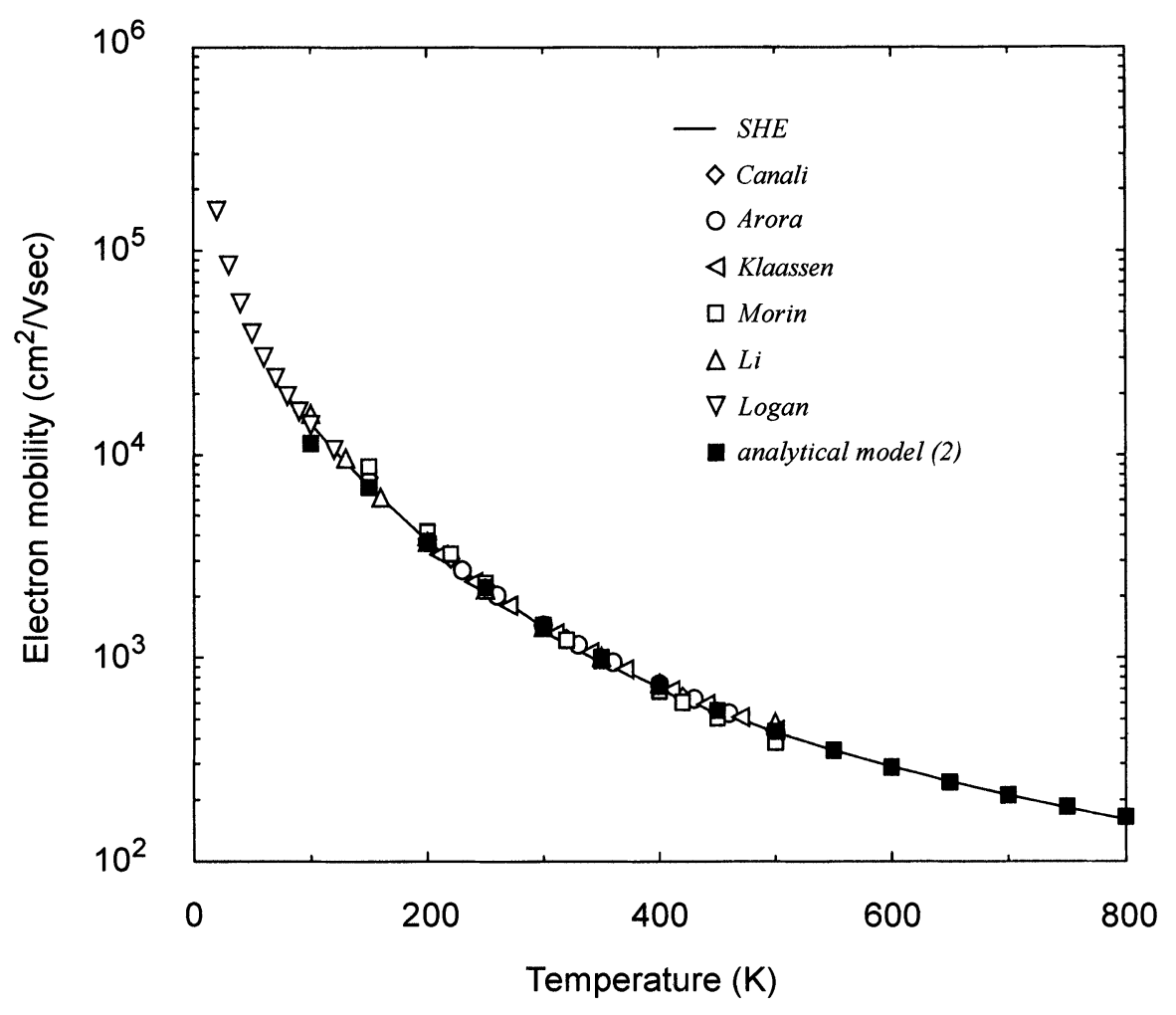

FIGURE 1 Intrinsic mobility computed with sHE, compared with the ones proposed by Logan [10], Canali [7], Arora [11], Klaassen [12], Li [4] and Morin [13]. The squares show the lattice mobility model (2) for electrons.

because of its influence at low temperatures and at high doping concentrations. Both the theoretical calculations and measurements indicate that at room temperature in silicon the dopant atoms are not completely ionized for concentrations higher than $10^{17} \mathrm{~cm}^{-3}[4]$. A physically-based relationship between the concentration of ionized dopant atoms and total doping concentration has been incorporated into the Boltzmann solver SHE, starting from a single-impurity-level theory and the description of the equilibrium carrier concentration in terms of Fermi statistics. This leads to the resistivity calculations shown in Figures 3 and 4 , where the inverse of the product between ionized-impurity concentration and carrier mobility has been calculated as a function of temperature at different total doping concentrations. The generalization introduced in the impurity-dependent contribution in (1) allows the model to correctly describe the temperature dependence of the carrier mobility at different doping concentrations, thus taking into account the partial-ionization effects. The model has finally been calibrated by comparison with experimental data for silicon for both electron and hole mobilities: the results are shown in Figures 5 and 6, demonstrating a good agreement for $T \geq 300 \mathrm{~K}$ in the whole range of doping concentrations. The agreement is still good below room temperature for a dopant concentration up to $10^{17} \mathrm{~cm}^{-3}$, whereas some discrepancy is found at higher concentrations. To this purpose, it is worth reminding that the numerical model had already been calibrated before, and the comparisons shown here have been carried out without further modifying the parameters; the accuracy of the model is 


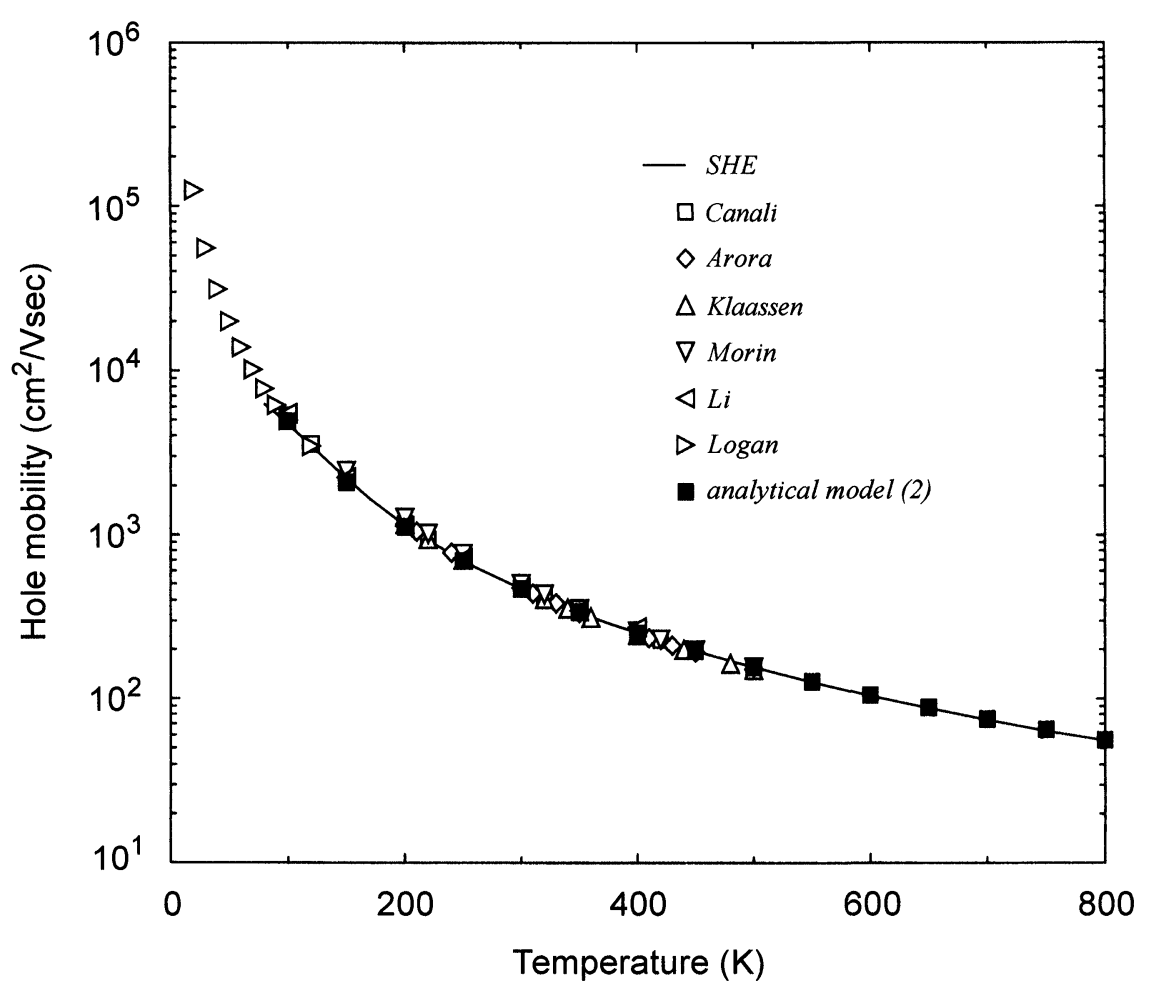

FIGURE 2 Intrinsic mobility computed with SHE, compared with the ones proposed by Logan [10], Canali [7], Arora [11], Klaassen [12], Li [14] and Morin [13]. The squares show the lattice mobility model (2) for holes.

TABLE I Parameters of model (2)

\begin{tabular}{lrr}
\hline Parameters & Electrons & Holes \\
\hline$\mu_{L 0}\left(\mathrm{~cm}^{2} /\right.$ Vsec $)$ & 1441.00 & 470.50 \\
$c$ & 0.07 & 0.00 \\
$\gamma$ & 2.45 & 2.16 \\
\hline
\end{tabular}

demonstrated by the curves of Figures 3 and 4, showing a comparison of the numerical model against experimentally-determined resistivities. In turn, the analytical model is able to reproduce the numerical one in the whole range of temperatures and doping concentrations shown in this paper. The experimental mobility curves of Figures 5 and 6 have been taken from [12] and originate from the resistivity data of [4]; however, no mention is made in [12] about the method by which mobility has been extracted from the resistivity data, in particular as far as the calculation of the effects of dopant freeze-out and bandgap narrowing are concerned. For this reason, it was not sensible to recalibrate the model, also in view of the fact that the present analysis aims at modeling high-temperature mobilities.

At high doping densities $\left(N \geq 10^{18} \mathrm{~cm}^{-3}\right)$ new physical effects become evident and must be taken into account. In particular, the impurityband formation alters the mobility shape around the impurity concentration of $10^{20} \mathrm{~cm}^{-3}$, for both electrons and holes, as already mentioned above. The nature of the mobility shape in the region of large impurity concentrations can qualitatively be explained as follows. First, as the impurity concentration increases, the screening effect due to the carrier concentration becomes relevant. Second, the periodic potential is altered by the distribution of 


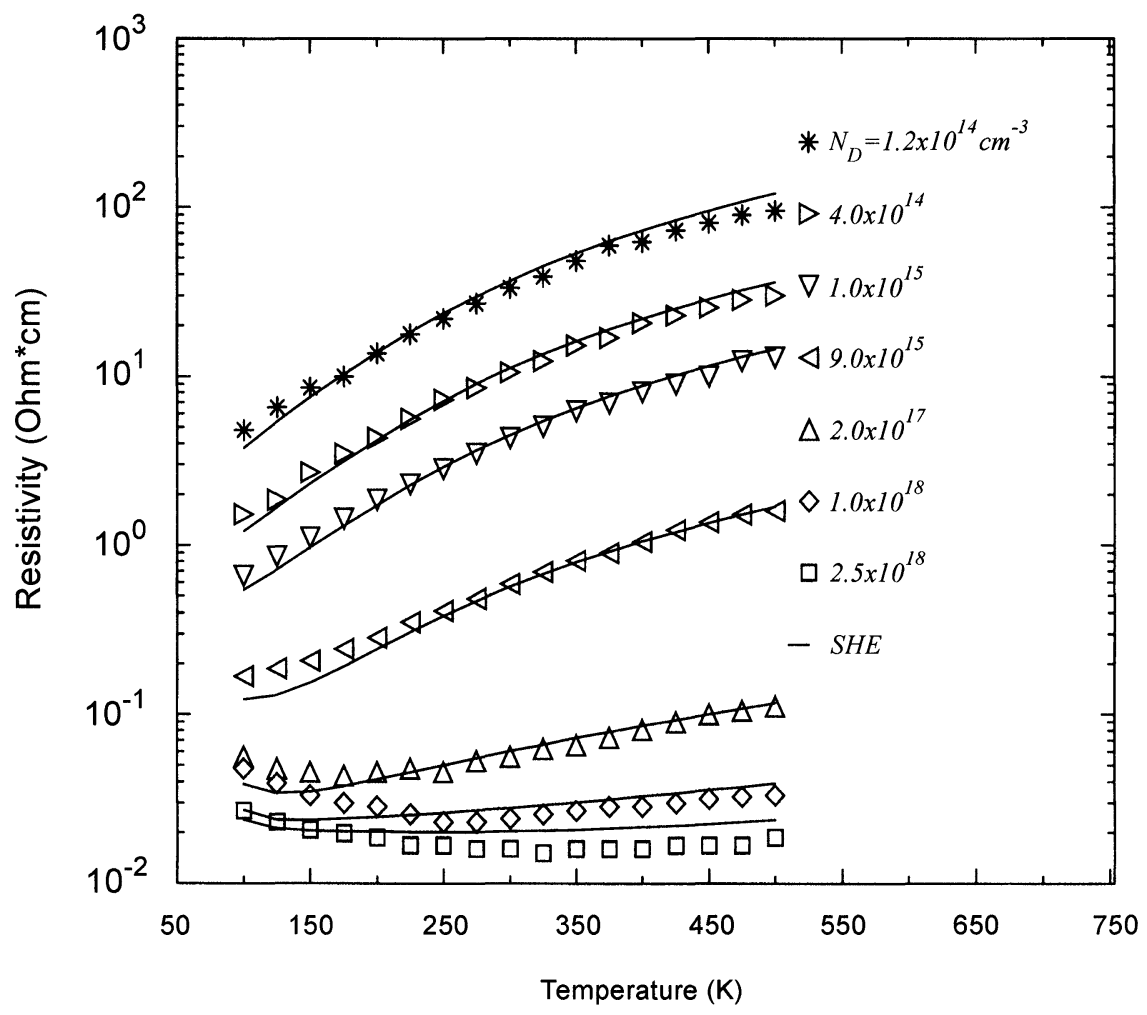

FIGURE 3 Electron resistivity as a function of temperature at different $N_{D}$, computed with the sHE code at the electric field of $2 \mathrm{kV} \mathrm{cm}^{-1}$, compared with $\mathrm{Li}$ [4].

impurity atoms in the lattice. Both effects increase with increasing concentrations. The latter effect is strongly related to the spreading of the states associated with the impurity atoms, that forms a quasi-continuous band of energies (the impurityband). Theoretical calculations showed that at a phosphorus concentration of $3 \cdot 10^{18} \mathrm{~cm}^{-3}$ the impurity band and the conduction band overlap to a significant extent: the conventional assumption of a delta-like distribution of impurity states fails [5]. Finally, the "step" shown by the mobility curve at about $4 \cdot 10^{20} \mathrm{~cm}^{-3}$ corresponds to the silicon degeneracy: the Fermi level enters the conduction band, and the two bands become indistinguishable.

The effects of a very high doping concentration were included here using the impurity-clustering approach in the Boltzmann solver [6]: in order to reproduce the mobility shape, an empirical clustering function has been worked out to fit the experiments, and then introduced into the Boltzmann solver. This approach accounts for the impurity-band formation without altering the numerical full-band structure adopted in SHE. It represents a sensible approach to the problem, as no experiments are available to date providing the density of states in heavily-doped semiconductors. The introduction of the clustering function in the impurity-scattering model has given very good results in the calculation of the majority-carrier low-field mobility by SHE, as can be seen in Figures 7 and 8. These calculations have been accounted for in the fitting procedure for the analytical model in order to study a wider range of doping concentrations and the related dependence on temperature. 


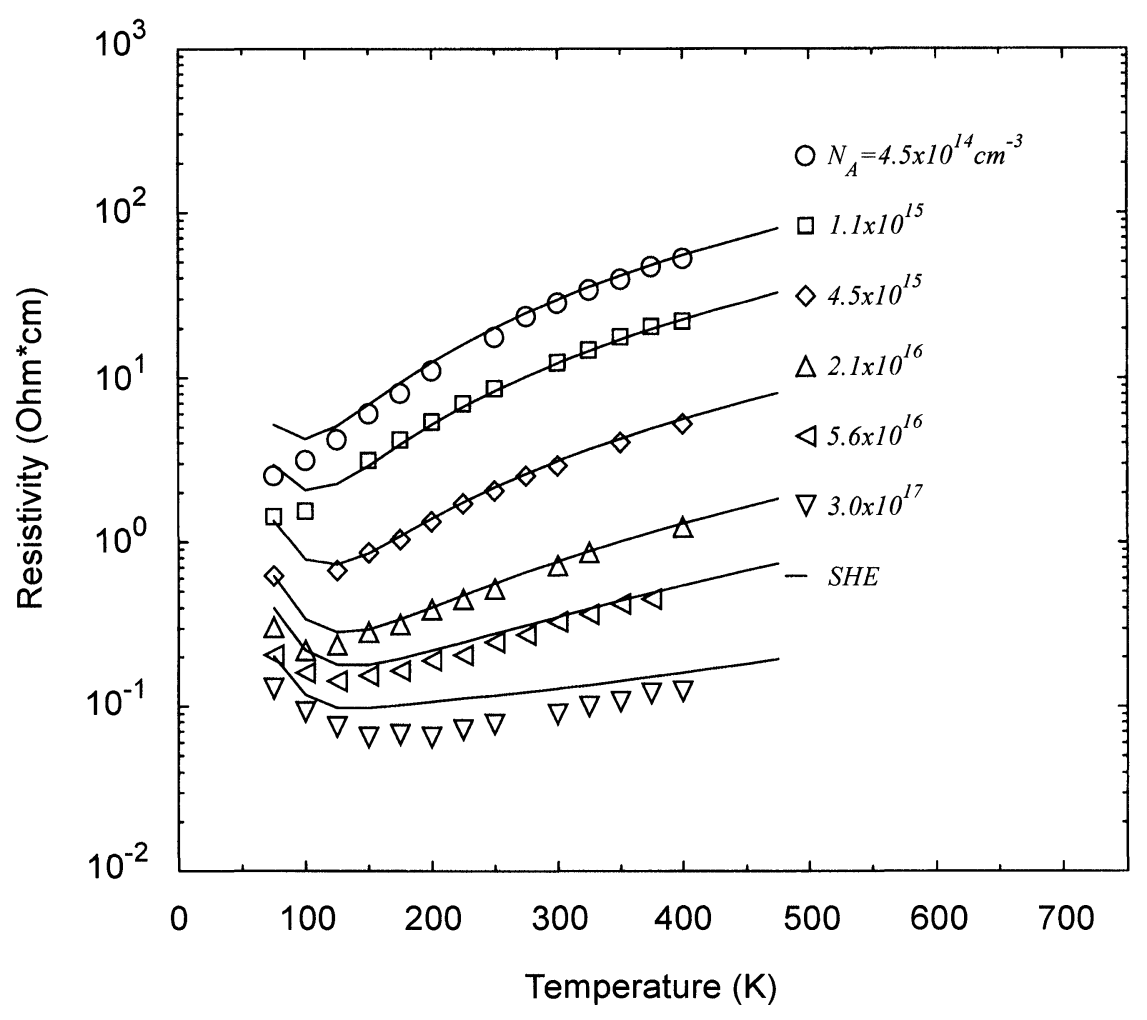

FIGURE 4 Hole resistivity as a function of temperature at different $N_{A}$, computed with the sHE code at the electric field of $2 \mathrm{kV} \mathrm{cm}^{-1}$, compared with Li [14].

\section{THE MAJORITY- AND MINORITY-BULK MOBILITY MODEL}

The modifications introduced in the ionizedimpurity scattering mechanism allow one to describe the low-field mobility of electrons and holes in a significant range of temperatures and doping concentrations, including the difference between majority and minority carriers. It is well known that the attractive- and repulsive-scattering potentials are different, this giving rise to a higher minority-carrier mobility in comparison with the majority-carrier one. This effect is not negligible, especially for holes as shown below, hence it must be accounted for as it influences the current level within the semiconductor devices. This is particularly important in the case of the BJT, where the current density is made of both the majority- and minority-carrier contributions.
Thanks to the generally-good agreement between the SHE model and the available experimental data, the SHE code has been used to generate a set of data for bulk mobility as a function of donor and acceptor concentrations and temperature. From the above data it was then possible to develop the new analytical mobility model for majority and minority carriers in bulk silicon.

As far as minority carriers are concerned, very few sets of measurements are available in the literature and, at least in the authors' knowledge, they are all at room temperature. The distinction between majority and minority carriers has been implemented by introducing suitable functions of both the acceptor and donor ion concentrations: as a matter of fact, the total concentration causes scattering and consequent mobility decrease, but the two species behave differently. These functions have been determined by the procedure 


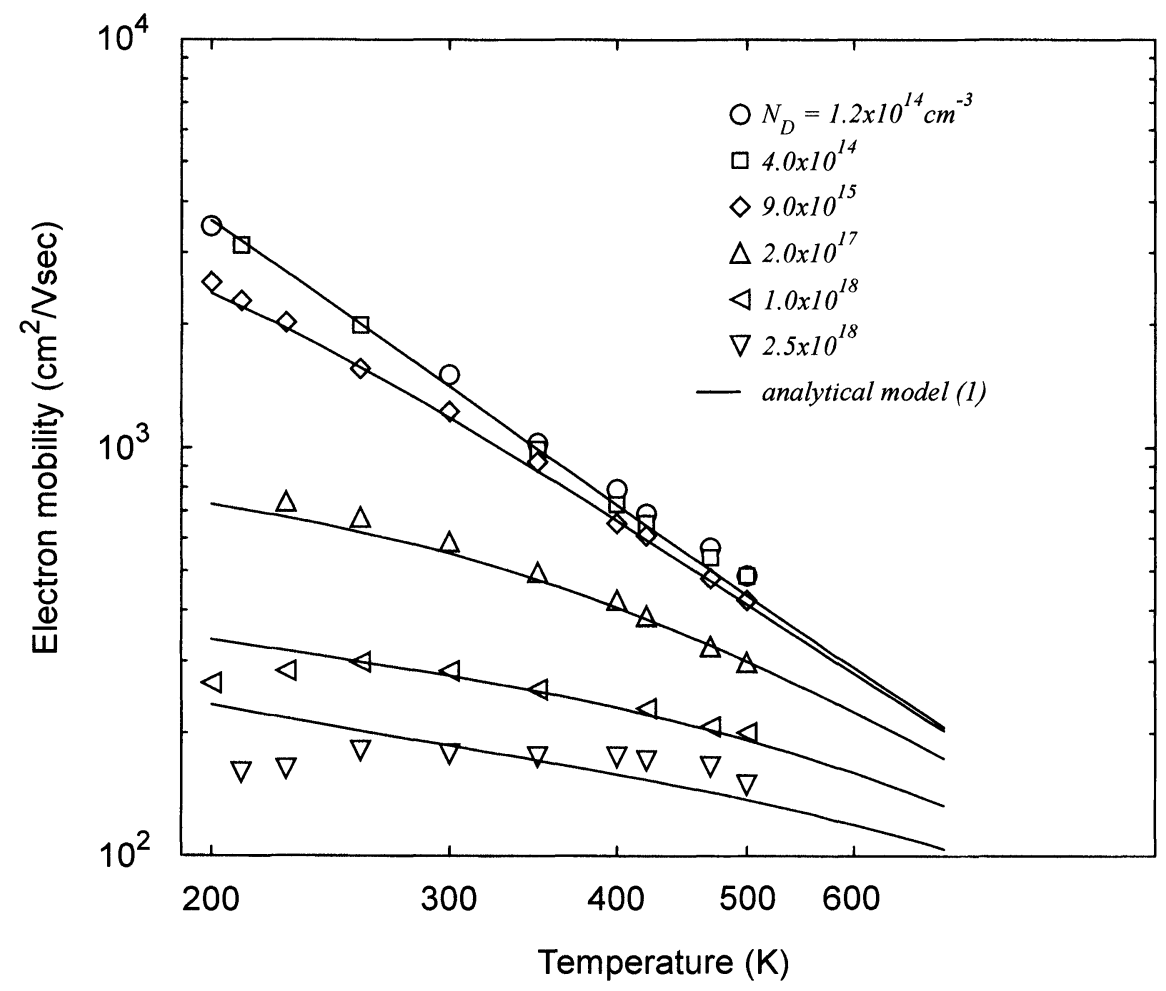

FIGURE 5 Electron mobility as a function of temperature at different $N_{D}$, computed with the model at the electric field of $2 \mathrm{kV} \mathrm{cm}^{-1}$, compared with $\mathrm{Li}$ [4].

depicted below. First, a set of minority-carrier mobility data has been generated by SHE and new sets of parameters have been defined to fit them. The results are shown in Figures 9 and 10. Then, a CAD-oriented approach has been followed to define a model for both majority and minority carriers. The model is made of a single continuous function, where both the donor and acceptor concentrations behave as independent variables, and the two different sets of previouslydetermined parameters are used. It reads

$$
\begin{aligned}
\mu_{b}\left(N_{D},\right. & \left.N_{A}, T\right) \\
= & \mu_{0}\left(N_{D}, N_{A}, T\right) \\
& +\frac{\mu_{L}(T)-\mu_{0}\left(N_{D}, N_{A}, T\right)}{1+\left(N_{D} / C_{r 1}(T)\right)^{\alpha_{1}}+\left(N_{A} / C_{r 2}(T)\right)^{\alpha_{2}}} \\
& -\frac{\mu_{1}\left(N_{D}, N_{A}, T\right)}{1+\left(N_{D} / C_{S 1}(T)+N_{A} / C_{s 2}(T)\right)^{-2}} .
\end{aligned}
$$

It is easily seen that the model is an extension of (1) where the contributions of $N_{A}$ and $N_{D}$ are combined to correctly predict the compensation effect, that is, when one of the doping species is dominant and defines the doping type, its contribution to the mobility becomes dominant with respect to the other. The expression for $\mu_{L}$ is still given by (2), while $\mu_{0}$ and $\mu_{1}$ have been reworked as

$$
\begin{aligned}
& \mu_{0}\left(N_{D}, N_{A}, T\right)=\frac{\mu_{0 d} N_{D}+\mu_{0 a} N_{A}}{N_{D}+N_{A}}, \\
& \mu_{1}\left(N_{D}, N_{A}, T\right)=\frac{\mu_{1 d} N_{D}+\mu_{1 a} N_{A}}{N_{D}+N_{A}} .
\end{aligned}
$$

The final fitting results and temperature dependencies for the model parameters are shown in Table II, with $T_{n}=T / 300$. 


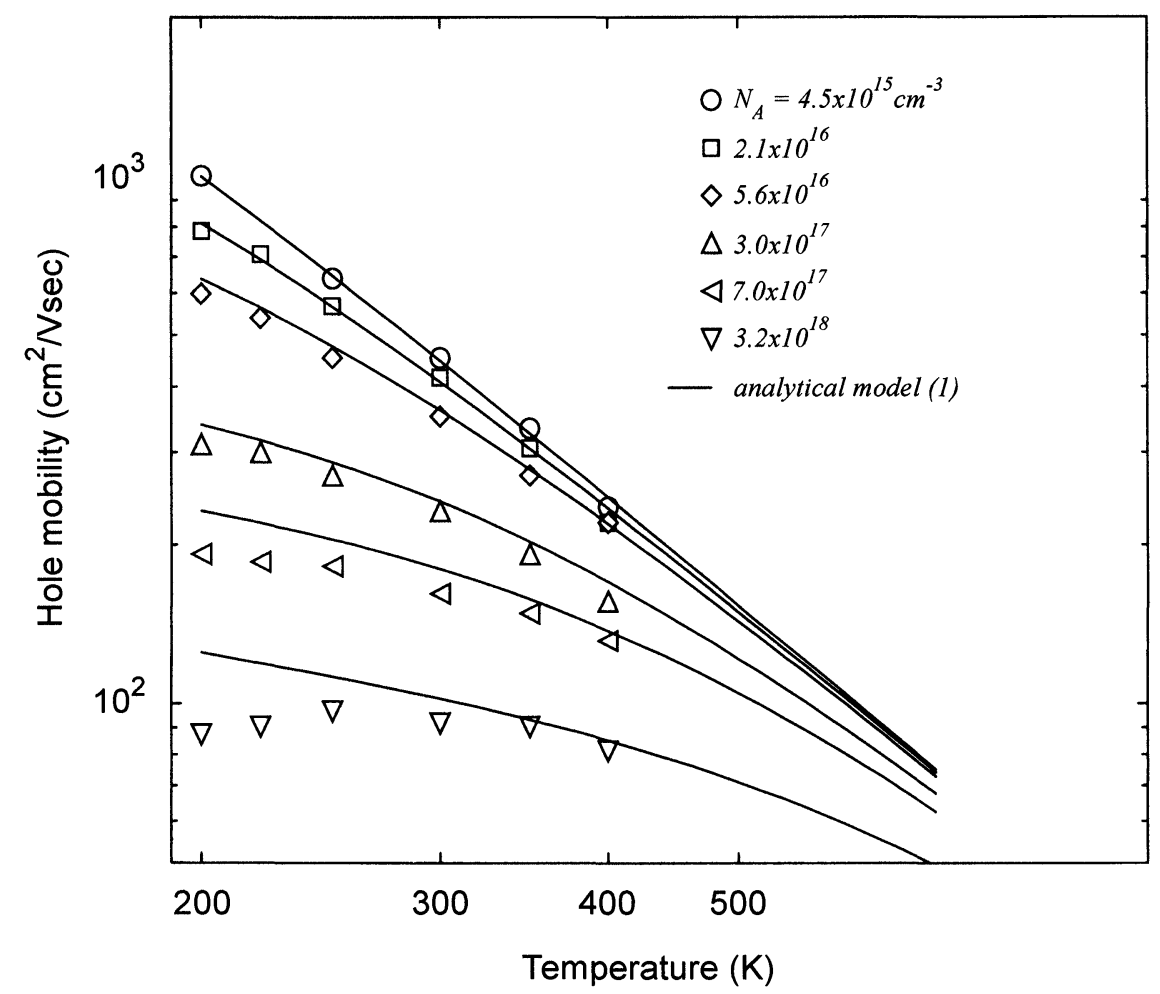

FIGURE 6 Hole mobility as a function of temperature at different $N_{A}$, computed with the model at the electric field of $2 \mathrm{kV} \mathrm{cm}^{-1}$, compared with $\mathrm{Li}[14]$.

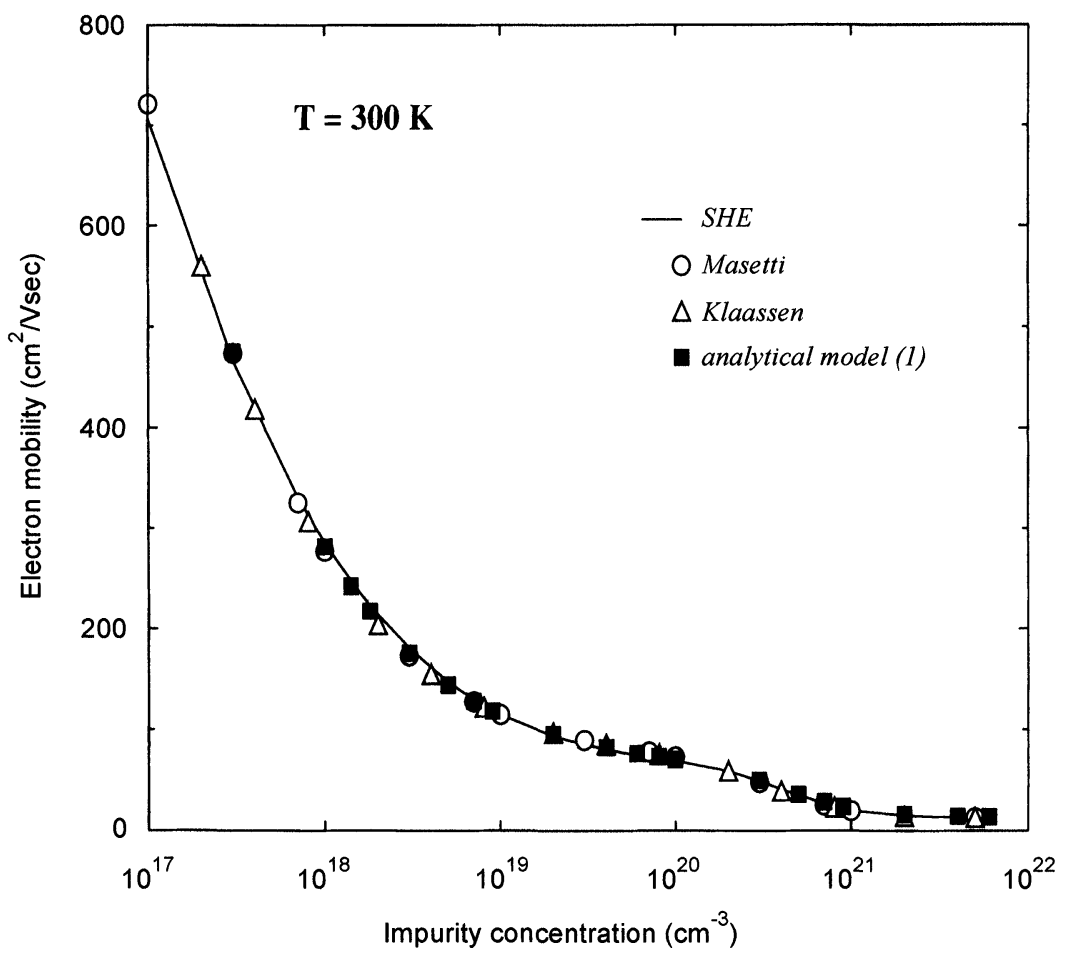

FIGURE 7 Electron mobility as a function of doping concentration, computed with the sHE code at the electric field of $2 \mathrm{kV} \mathrm{cm}^{-1}$, compared with Masetti [3] and Klaassen [12]. Squares show the doping-dependent mobility model for electrons (1). 


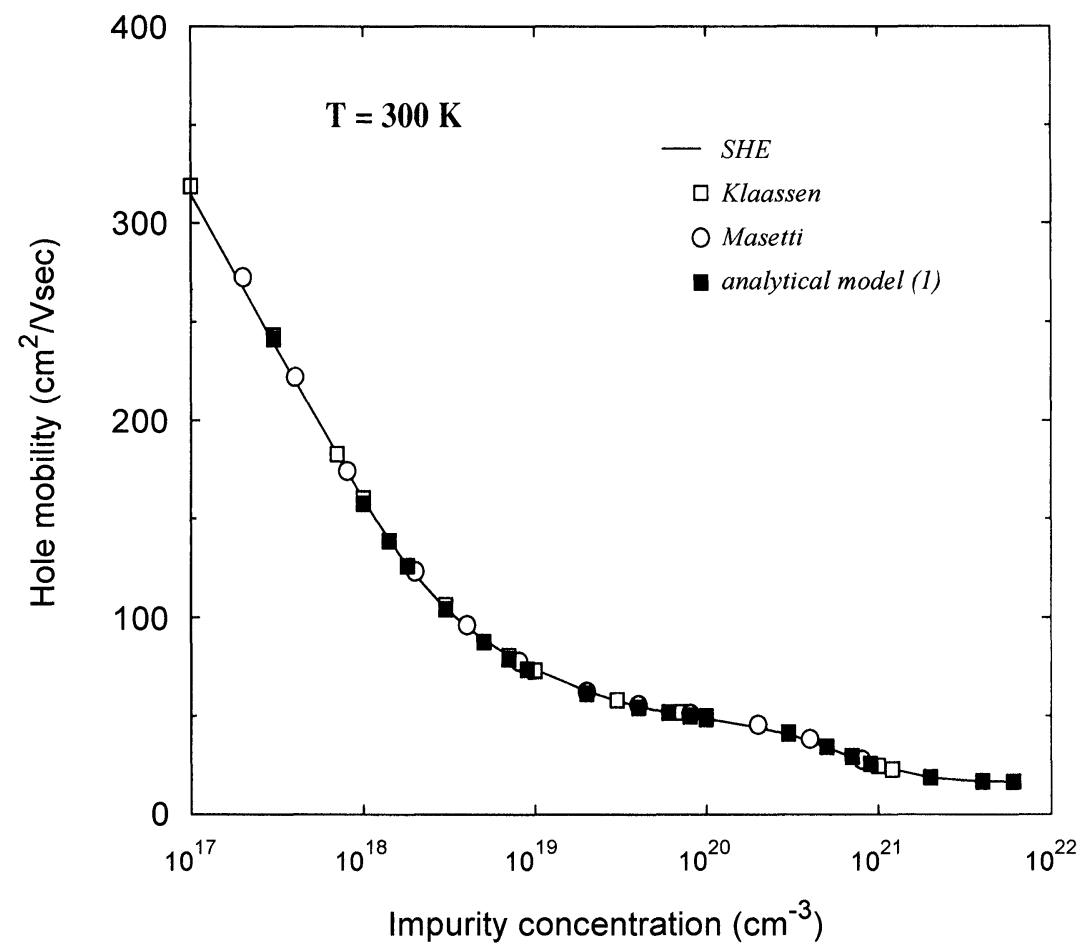

FIGURE 8 Hole mobility as a function of doping concentration, computed with the sHE code at the electric field of $2 \mathrm{kV} \mathrm{cm}^{-1}$, compared with Masetti [3] and Klaassen [12]. Squares show the doping-dependent mobility model for holes (1).

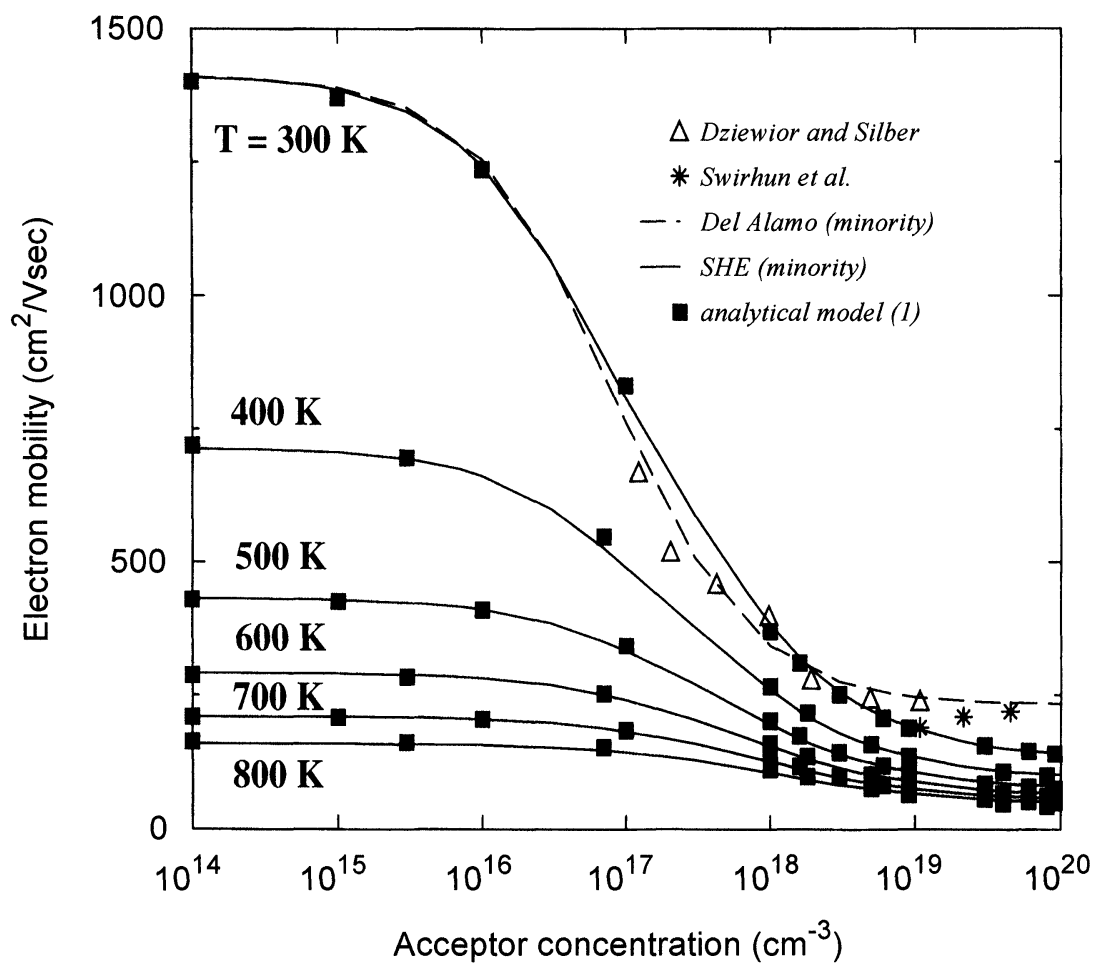

FIGURE9 Minority-electron mobility as a function of doping concentration, computed with the sHE code at different temperatures and with an electric field of $2 \mathrm{kV} \mathrm{cm}^{-1}$, compared with Del Alamo's model [15] and experiments by Dziewior and Silber [16] and Swirhun et al. [17]. Open circles show the minority-carrier mobility model for electrons. 


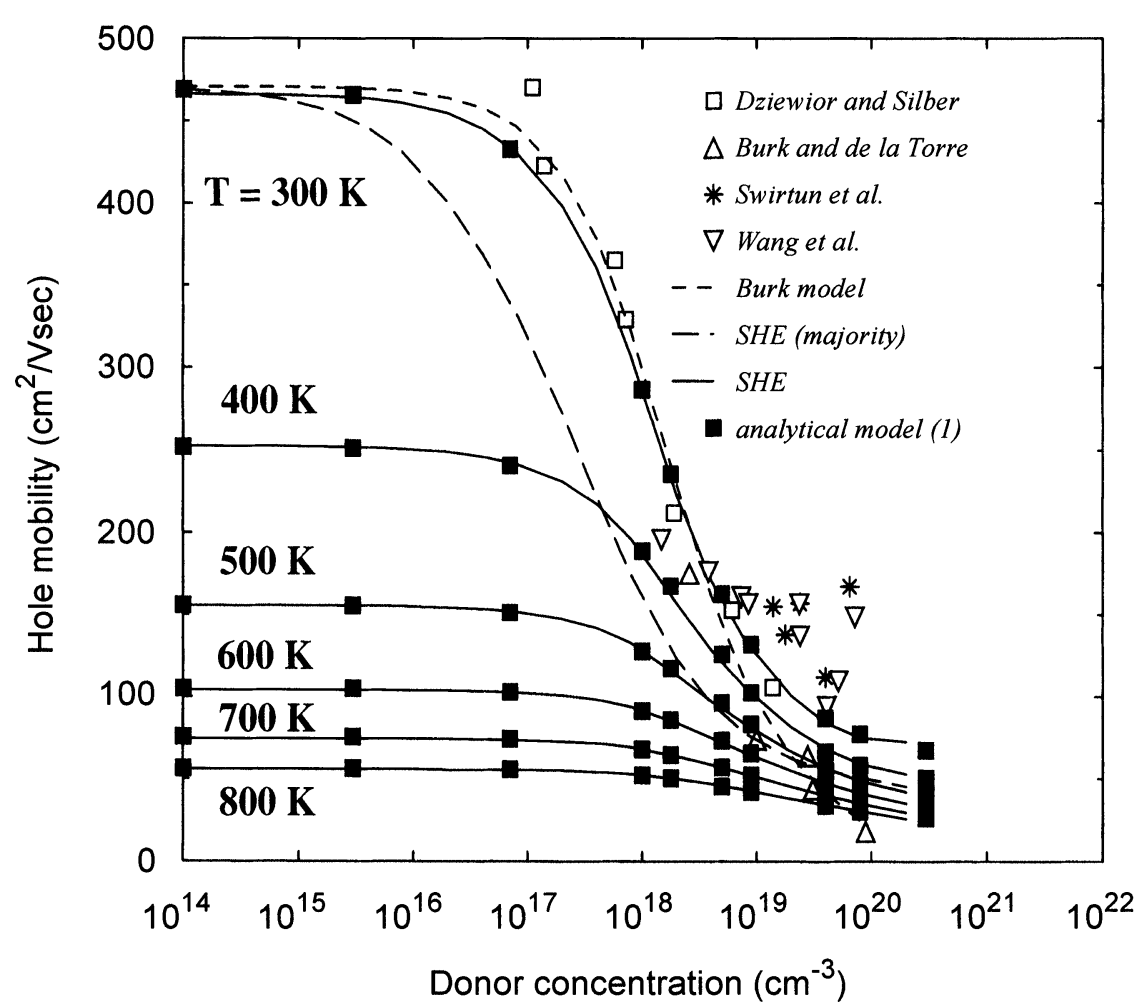

FIGURE 10 Minority-hole mobility as a function of doping concentration, computed with the sHE code at different temperatures and with an electric field of $2 \mathrm{kV} \mathrm{cm}^{-1}$, compared with experiments by Dziewior and Silber [16], Burk and de la Torre [18], Swirhun et al. [19] and Wang et al. [20]. Open circles show the minority-carrier mobility model for holes.

TABLE II Parameters of model (3)

\begin{tabular}{lll}
\hline Parameters & \multicolumn{1}{c}{ Electrons } & \multicolumn{1}{c}{ Holes } \\
\hline$\mu_{0 d}\left(\mathrm{~cm}^{2} / \mathrm{Vsec}\right)$ & $62.2 \cdot T_{n}^{-1.3}$ & $90.0 \cdot T_{n}^{-1.3}$ \\
$\mu_{0 a}\left(\mathrm{~cm}^{2} / \mathrm{Vsec}\right)$ & $132.0 \cdot T_{n}^{-1.3}$ & $44.0 \cdot T_{n}^{-0.8}$ \\
$\mu_{1 d}\left(\mathrm{~cm}^{2} / \mathrm{Vsec}\right)$ & $48.6 \cdot T_{n}^{-0.7}$ & $28.2 \cdot T_{n}^{-2.0}$ \\
$\mu_{1 a}\left(\mathrm{~cm}^{2} / \mathrm{Vsec}\right)$ & $73.5 \cdot T_{n}^{-1.25}$ & $28.2 \cdot T_{n}^{-0.2}$ \\
$C_{r 1}\left(\mathrm{~cm}^{-3}\right)$ & $8.30 \cdot 10^{16} \cdot T_{n}^{3.65}$ & $1.30 \cdot 10^{18} \cdot T_{n}^{2.2}$ \\
$C_{r 2}\left(\mathrm{~cm}^{-3}\right)$ & $1.22 \cdot 10^{17} \cdot T_{n}^{2.65}$ & $2.45 \cdot 10^{17} \cdot T_{n}^{3.1}$ \\
$C_{s 1}\left(\mathrm{~cm}^{-3}\right)$ & $4.00 \cdot 10^{20}$ & $1.1 \cdot 10^{18} \cdot T_{n}^{6.2}$ \\
$C_{s 2}\left(\mathrm{~cm}^{-3}\right)$ & $7.00 \cdot 10^{20}$ & $6.10 \cdot 10^{20}$ \\
$\alpha_{1}$ & 0.68 & 0.77 \\
$\alpha_{2}$ & 0.72 & 0.719 \\
\hline
\end{tabular}

\section{THE SATURATION-VELOCITY EFFECT}

The high electric-field effect was preliminarily analyzed using the Boltzmann solver: in Figures 11 and 12 the drift velocity has been calculated at different temperatures up to $5 \cdot 10^{5} \mathrm{~V} / \mathrm{cm}$ and compared with an empirical model [7]. Monte Carlo data were available for holes at room temperature [8], and good agreement has been found also in this case. No measurements of the saturation velocity are available at significant impurity concentrations. In the new analytical model, the carrier saturation velocity $v_{s}$ is assumed independent of the impurity concentration and transverse electric field. In the electron case, the temperature dependence of $v_{s}$ is given by [7]:

$$
v_{s}^{\mathrm{el}}(T)=\frac{v_{s 0}^{\mathrm{el}}}{1+\vartheta \exp (T / 600)},
$$

where $v_{s 0}^{\mathrm{el}}=2.4 \cdot 10^{7} \mathrm{~cm} / \mathrm{s}$ and $\vartheta=0.8$. This empirical function was worked out starting from measurements, whereas no similar experiments are available for the hole-saturation velocity. For 


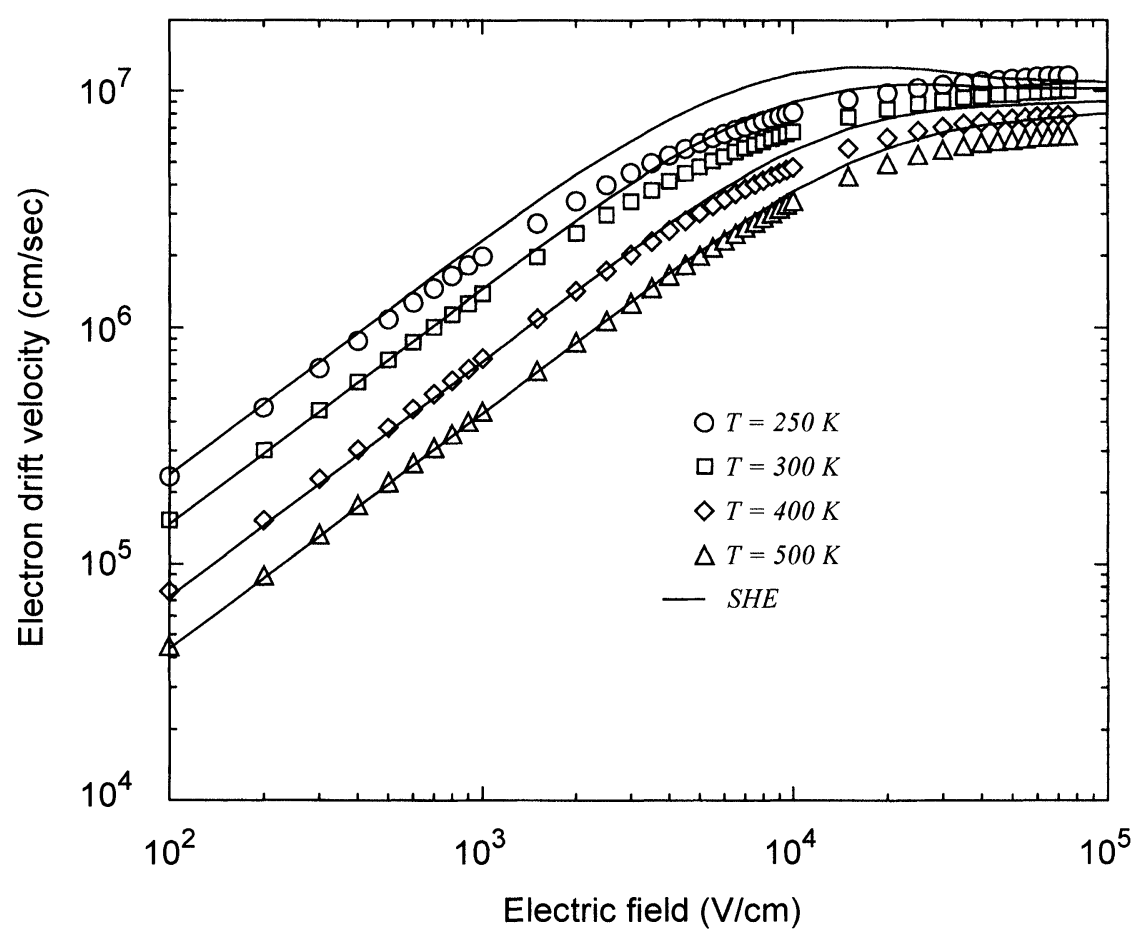

FIGURE 11 Electron-drift velocity calculated by SHE at different temperatures and compared with the empirical model by Jacoboni et al. [7].

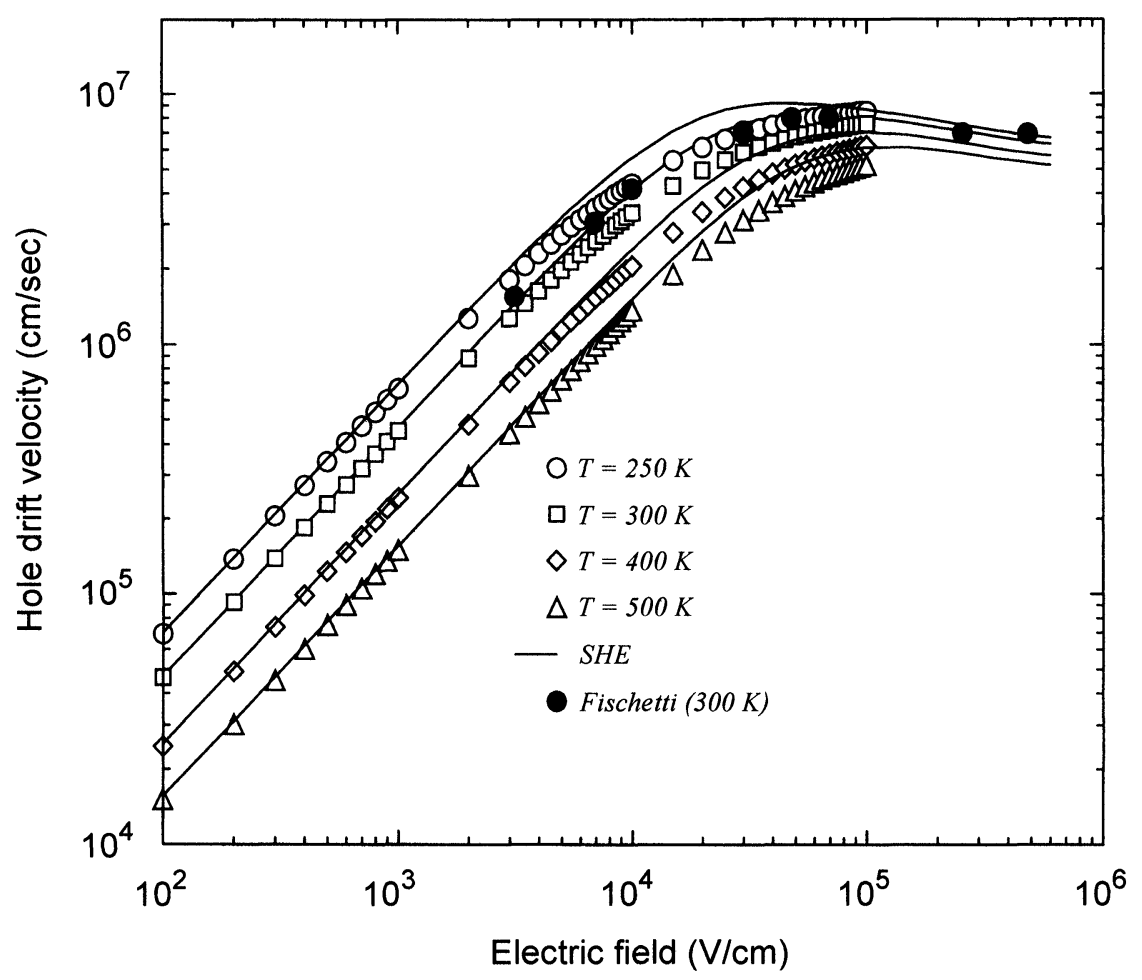

FIGURE 12 Hole-drift velocity calculated by SHE at different temperatures and compared with Monte Carlo data by Fischetti and Laux [8] and with the empirical model by Jacoboni et al. [7]. 
TABLE III Parameters of model (8)

\begin{tabular}{lll}
\hline Parameters & Electrons & Holes \\
\hline$b_{1}$ & $2.1 \cdot T_{n}^{-0.2}$ & $2 \cdot T_{n}^{-0.2}$ \\
$b_{2}$ & 2 & 0.6 \\
$b_{3}$ & 3 & 0.6 \\
$b_{h 1}$ & - & $0.15 \cdot T_{n}$ \\
$b_{h 2}$ & - & 0.8 \\
$b_{h 3}$ & - & 0.8 \\
$C_{t 1}\left(\mathrm{~cm}^{-3}\right)$ & $10^{19}$ & $8 \cdot 10^{17}$ \\
$C_{t 2}\left(\mathrm{~cm}^{-3}\right)$ & $10^{19}$ & $10^{19}$ \\
\hline
\end{tabular}

the latter, the following expression has been used:

$$
v_{s}^{\mathrm{ho}}(T)=v_{s 0}^{\mathrm{ho}}(T / 300)^{-\eta}
$$

with $v_{s 0}^{\text {ho }}=9.1 \cdot 10^{6} \mathrm{~cm} / \mathrm{s}$ and $\eta=0.4$.

Under the assumption that the saturation velocity in devices is the same as in bulk silicon, the analytical model (3) has been extended in a conventional way in order to incorporate the high electric-field effects:

$$
\mu_{B}\left(E, N_{A}, N_{D}, T\right)=\frac{\mu_{b}}{\left[1+\left(\mu_{b} E / v_{s}\right)^{\beta+\beta_{h}}\right]^{1 / \beta}},
$$

where $v_{s}$ is given by (6) for electrons and (7) for holes, and $\mu_{b}$ is given by (3). The model has been compared with the SHE calculations relative to a bulk structure. It is worth mentioning that the asymptotic expressions for electrons and holes at very high electric fields are different: while the electron mobility is well described by a law proportional to $1 / E$, the hole mobility shows a stronger dependence on the electric field, behaving as $1 / E^{1+\beta h / \beta}$. The difference between the electronand hole-mobility behaviors can be ascribed to the strongly different shape of the conduction and valence bands at high energies.

Using a similar procedure as the one depicted for (3), the temperature and doping dependencies of $\beta$ and $\beta_{h}$ have been incorporated into the

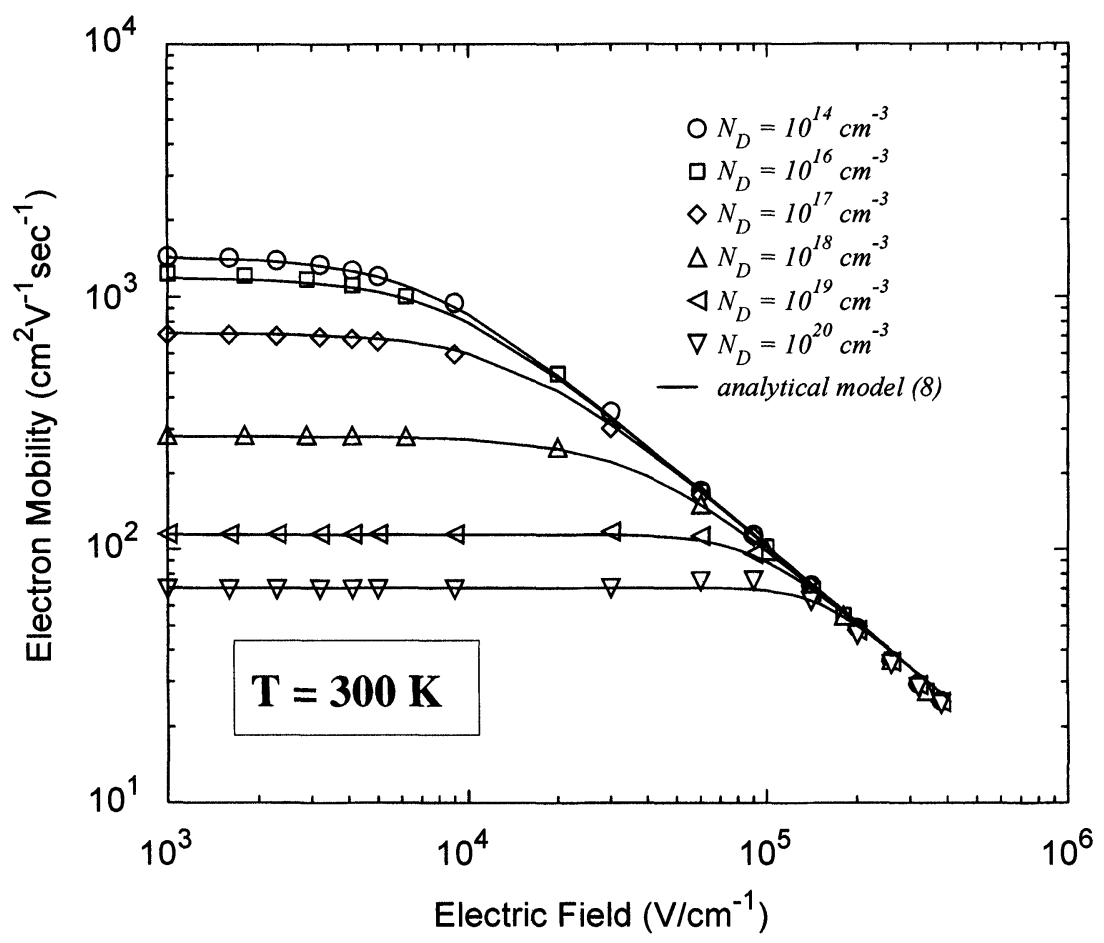

FIGURE 13 Majority-electron bulk mobility computed with the analytical model (8) and with sHE at $T=300 \mathrm{~K}$. 


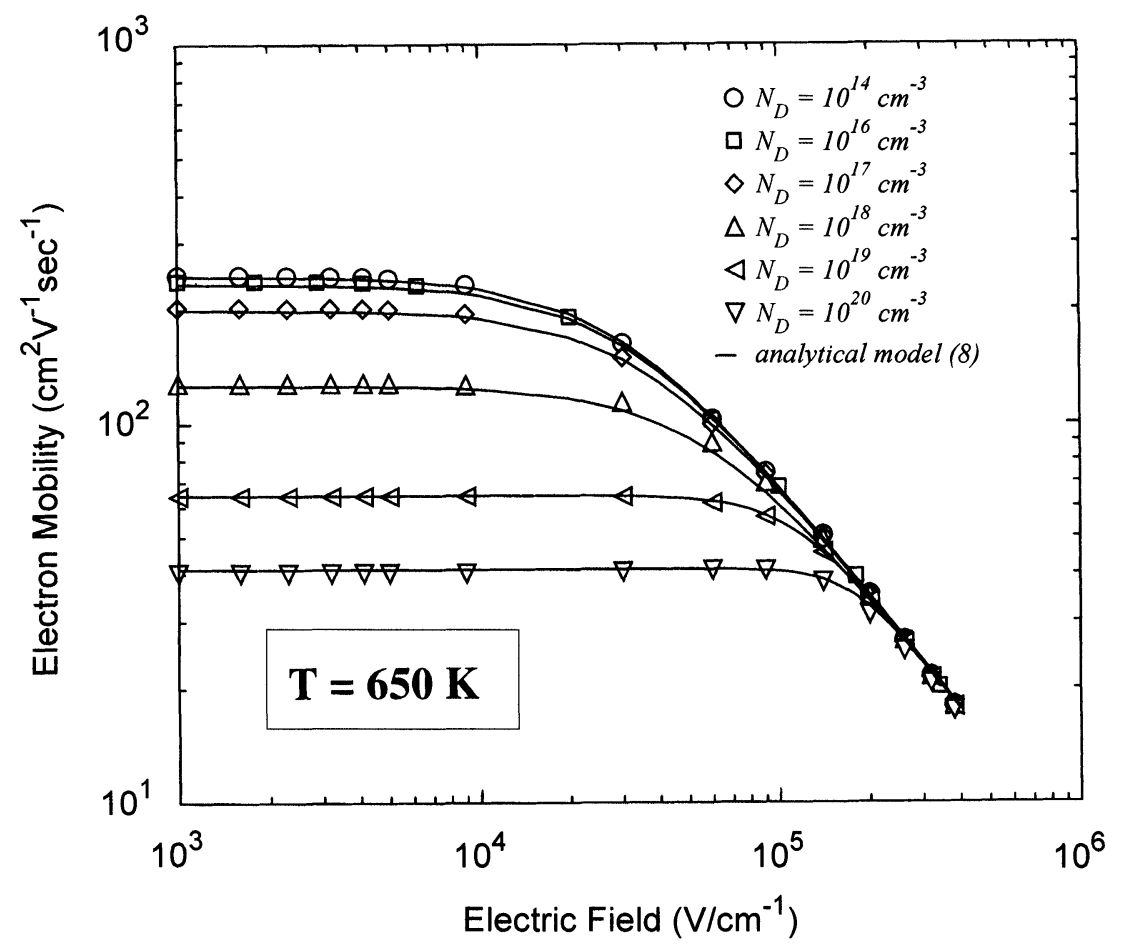

FIGURE 14 Majority-electron bulk mobility computed with the analytical model (8) and with sHE at $T=650 \mathrm{~K}$.

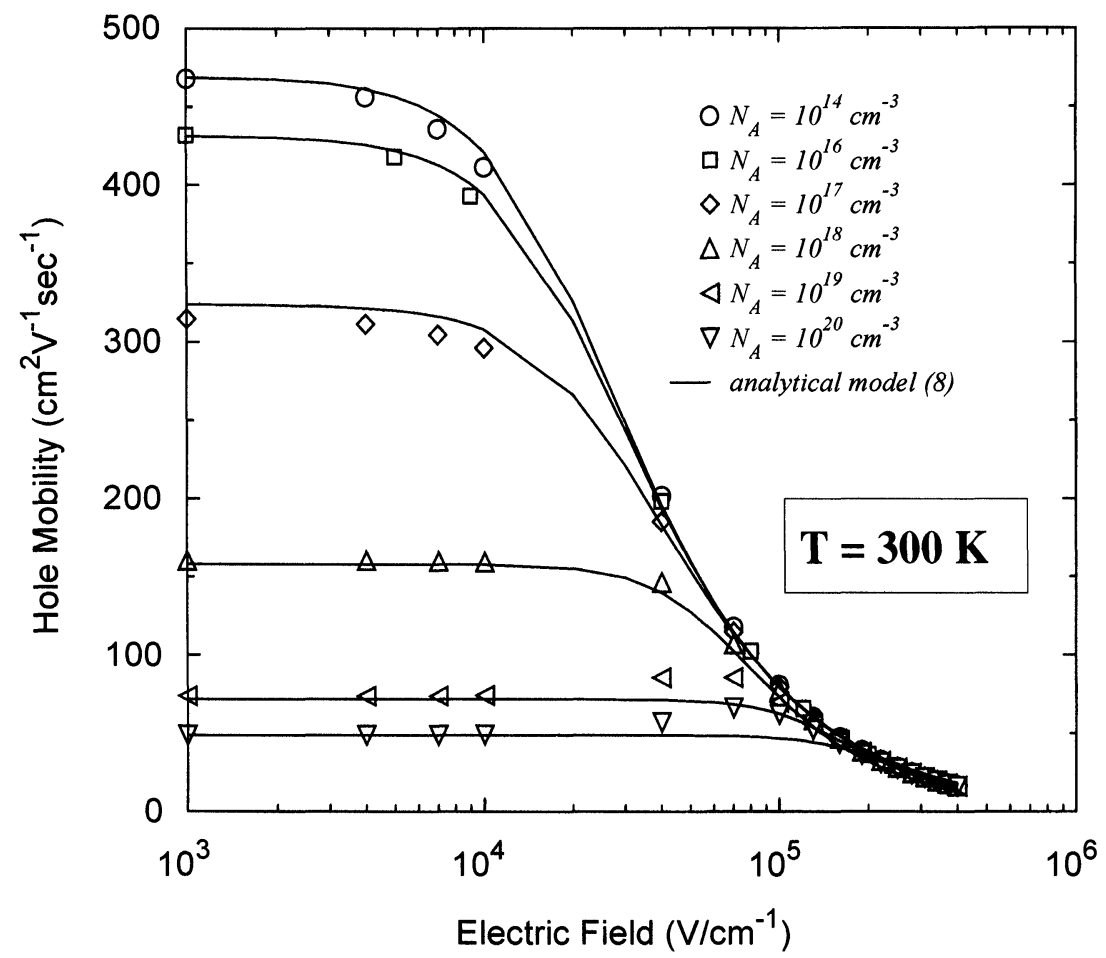

FIGURE 15 Majority-hole bulk mobility computed with the analytical model (8) and with SHE at $T=300 \mathrm{~K}$. 


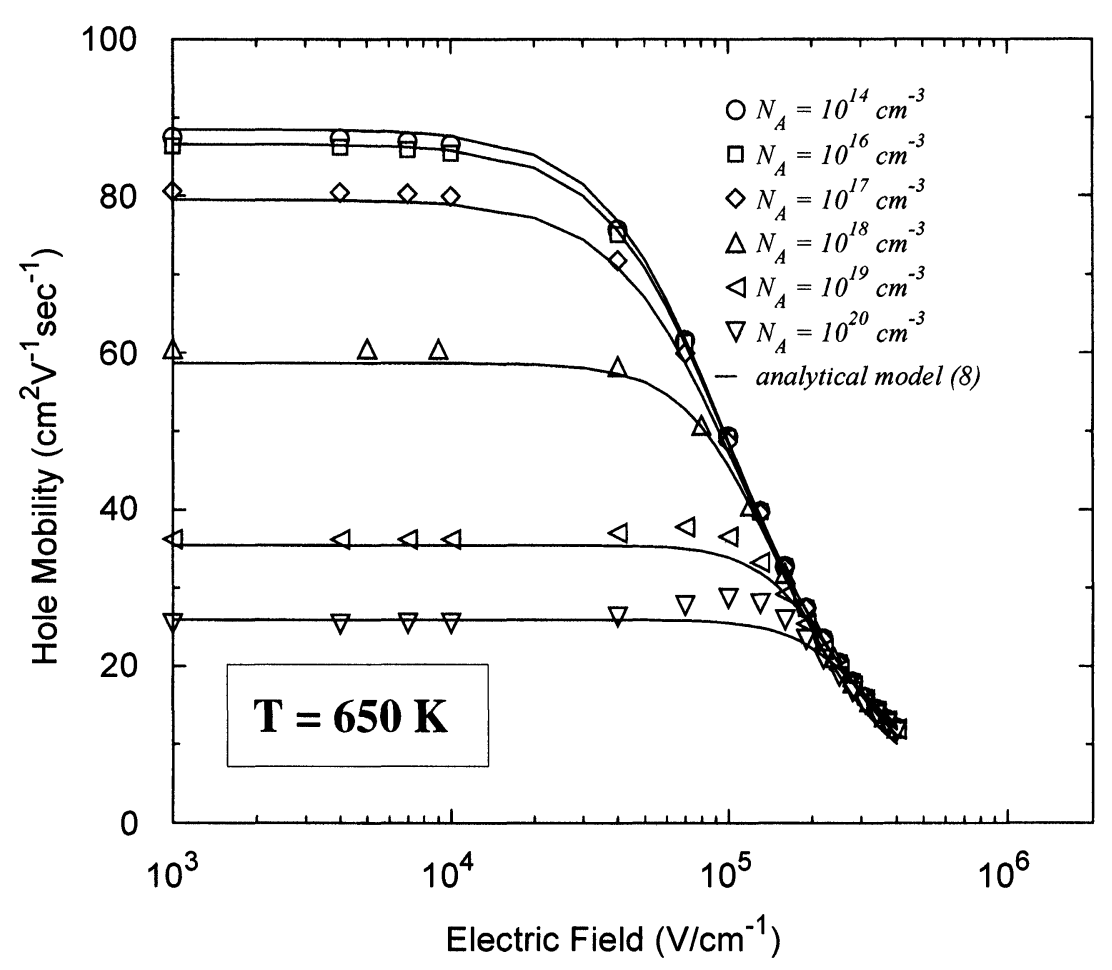

FIGURE 16 Majority-hole bulk mobility computed with the analytical model (8) and with sHE at $T=650 \mathrm{~K}$.

following analytical functions reproducing the SHE data:

$$
\begin{aligned}
\beta\left(N_{A}, N_{D}, T\right)= & b_{1}+\frac{b_{2}}{1+\left(C_{t 1} / N_{A}\right)^{2}} \\
& +\frac{b_{3}}{1+\left(C_{t 2} / N_{D}\right)^{2}}, \\
\beta_{h}\left(N_{A}, N_{D}, T\right)= & b_{h 1}+\frac{b_{h 2}}{1+\left(C_{t 1} / N_{A}\right)^{2}} \\
& +\frac{b_{h 3}}{1+\left(C_{t 2} / N_{D}\right)^{2}} .
\end{aligned}
$$

The physical arguments that explain these dependencies are again related to the non-parabolic energy band shape and to the band deformation due to the overlapping of the impurity band at very high doping concentrations. The parameters appearing in the expressions of $\beta$ and $\beta_{h}$ are reported in Table III, with $T_{n}=T / 300$.

The mobility curves for majority carriers, resulting from the application of the complete analytical model (8) at different doping concentrations, temperatures, and electric fields, have been gathered in Figures 13, 14 for electrons and Figures 15, 16 for holes, and compared with the SHE calculations.

\section{CONCLUSIONS}

A physically-based, analytical mobility model for silicon has been worked out, incorporating the distinction between majority and minority carriers, the partial-ionization and high-field effects, and the dependencies on the lattice temperature up to $800 \mathrm{~K}$. At room temperature the experimental results of [3] are well reproduced in the whole range of doping concentrations. At different temperatures a number of extensions have been taken into account in the analytical model, such that the model agrees well with a large amount of experimental data that are available in the literature for majority carriers, up to $500 \mathrm{~K}$. The 
model has then been extended up to $800 \mathrm{~K}$ by validation against numerical data obtained from a Boltzmann solver based on first-principle physical models. The high-field mobility model has been worked out and compared as well with numerical data obtained from the Boltzmann solver.

The model is especially suited for device simulation purposes, because the carrier mobilities are provided as analytical functions of local variables: donor and acceptor concentrations, electric field and temperature. The model has now been implemented in the 2-D numerical device simulator DESSIS ${ }^{(}$[9], and work is currently in progress for further testing and validation.

\section{References}

[1] Ventura, D., Gnudi, A. and Baccarani, G. (1995). "A Deterministic Approach to the Solution of the BTE in Semiconductors", La Rivista del Nuovo Cimento, 18(6), $1-33$.

[2] Reggiani, S., Vecchi, M. C. and Rudan, M., "Investigation on Electron and Hole Transport Properties Using the Full-Band Spherical-Harmonics Expansion Method", IEEE Trans. on ED, 45(9), 2010-2017, September, 1998.

[3] Masetti, G., Severi, M. and Solmi, S., "Modeling of carrier mobility against carrier concentration in arsenic-, phosphorus-, and boron-doped silicon", IEEE Trans. on ED, 30(7), 764-769, July, 1983.

[4] Li, S. S. and Thurber, W. R. (1977). "The dopant density and temperature dependence of electron mobility and resistivity in $n$-type silicon", Solid State Electronics, 20, $609-616$.

[5] Kleppinger, D. D. and Lindholm, F. A. (1971). "Impurity concentration dependent density of states and resulting Fermi level for silicon", Solid State Electronics, 14, 407-416.

[6] Klaassen, D. B. M. (1992). "A unified mobility model for device simulation-I. Model equations and concentration dependence", Solid State Electronics, 35(7), 953-959.

[7] Jacoboni, C., Canali, C., Ottaviani, G. and Alberigi Quaranta, A. (1977). "A review of some charge transport properties of silicon", Solid State Electronics, 20, 77-89.

[8] Fischetti, M. V. and Laux, S. E. (1988). "Monte-Carlo Analysis of Electron Transport in Small Semiconductor Devices Including Band-Structure and Space-Charge Effects", Phys. Rev. B, 38, 9721.

[9] ISE Integrated Systems Engineering AG, ISE TCAD Manuals, V. 5.0, Part 14: DESSIS, 1998.

[10] Logan, R. A. and Peters, A. J., "Impurity effects upon mobility in silicon", Journal of Applied Physics, 31(1), 122-124, January, 1960

[11] Arora, N. D., Hauser, J. R. and Roulston, D. J. "Electron and hole mobilities in silicon as a function of concentration and temperature", IEEE Transactions on Electron Devices, ED-29(2), 292-295, February, 1982.
[12] Klaassen, D. B. M. (1992). "A unified mobility model for device simulation-II. Temperature dependence of carrier mobility and lifetime", Solid State Electronics, 35(7), $961-967$.

[13] Morin, F. J. and Maita, J. P., "Electrical properties of silicon containing arsenic and boron", Physical Review, 96(1), 28-35, October, 1954.

[14] Li, S. S. (1978). "The dopant density and temperature dependence of hole mobility and resistivity in borondoped silicon", Solid State Electronics, 21, 1109-1117.

[15] Del Alamo, J., Swirhun, S. E. and Swanson, R. M. (1985) "Measurement and modeling of minority carrier transport in heavily-doped silicon", Solid-State Electronics, 28, 47-52.

[16] Dziewior, J. and Silber, D., "Minority-carrier diffusion coefficients in highly-doped silicon", Appl. Phys. Lett., 35, 170-172, July, 1979.

[17] Swirhun, S. E., Kwark, Y. H. and Swanson, R. M. (1986). IEDM Tech. Dig., p. 24.

[18] Burk, D. E. and De La Torre, V., "An empirical fit to minority hole mobilities", IEEE Electron Device Letters, 5(7), 231-233, July, 1984.

[19] Swirhun, S. E., Del Alamo, J. A. and Swanson, R. M. "Measurement of hole mobility in heavily doped $n$-type silicon", IEEE Electron Device Letters, 7(3), 168-171, March, 1986.

[20] Wang, C. H., Misiakos and Neugroschel, A., "Minoritycarrier transport parameters in n-type silicon", IEEE Trans. on ED, 37(5), 1314-1322, May, 1990.

\section{Authors' Biographies}

Susanna Reggiani received the degree in Electrical Engineering from the University of Bologna, Italy, in 1997. Since April 1997 she has been working at the Department of Electronics (DEIS) of the same University in the field of the numerical simulation of semiconductor devices. She is currently attending the Ph.D. course in Electrical Engineering at the University of Bologna.

Marina Valdinoci received the degree in Electrical Engineering from the University of Bologna, Italy, in 1991. In 1992, profiting by a fellowship provided by SGS-Thomson Microelectronics and Co.Ri.M.Me., she started working at the Department of Electronics (DEIS) of the same University, investigating the performance of gold- and platinum-doped power bipolar devices. In 1997 she received the Ph.D. in Electrical Engineering from the University of Bologna, on the subject of the numerical analysis of amorphous and polycrystalline-silicon devices. Her present interests are in the modeling of ESD phenomena in silicon. 
Luigi Colalongo received the degree in Electrical Engineering from the University of Bologna, Italy, in 1992. In 1993, profiting by a fellowship provided by SGS-Thomson Microelectronics and Co.Ri.M.Me., he started working at the Department of Electronics (DEIS) of the same University, investigating the numerical methods for the time-dependent solution of the semiconductor equations. In 1998 he received the Ph.D. in Electrical Engineering from the University of Trento, Italy, on the subject of the CAD and characterization of semiconductor sensors of the ISFET and LAPS type. In 1999 he has become Research Assistant at the Faculty of Engineering of the University of Brescia, Italy.

Massimo Rudan received a degree in Electrical Engineering in 1973 and a degree in Physics in 1976, both from the University of Bologna, Italy. He joined the Department of Electronics (DEIS) of the University of Bologna in 1975. From 1978 he has been teaching a course of Quantum Electronics in the Faculty of Engineering of the same University. Since 1983 he has been working in a group involved in numerical analysis of semiconductor devices. In 1986 he has been a visiting scientist at the IBM Thomas J. Watson Research Center at Yorktown Heights, NY, studying the discretization techniques for the higher-order moments of the Boltzmann Equation. In 1990, he was appointed Full Professor of Microelectronics at the University of Bologna.
Giorgio Baccarani received a degree in Electrical Engineering in 1967 and a degree in Physics in 1969, both from the University of Bologna, Italy, where he currently is a full professor in charge of an annual Course on Digital Systems Design. Prof. Baccarani has devoted his research work to various aspects of Microelectronics, including processing technology, device physics and characterization, current transport in semiconductor materials and devices, submicron MOSFET scaling and optimization, numerical analysis of semiconductor devices, analog design and, more recently, cognitive systems architectures. He authored or coauthored about 130 papers, and his activity is the result of an extensive cooperation with $\mathrm{Na}$ tional, European and American Companies, as well as with a number of research Institutions in Italy and abroad. He was a visiting scientist at the ATT-Bell Telephone Laboratories in Murray-hill, NJ in 1969-70; at the IBM T. J. Watson Research Center in Yorktown Heights in 1981-82, 1983 and in 1988 . He is currently chairman of the Scientific Boards of the National Institute for Microelectronics Technology (CNR-IMETEM) and of the Joint Laboratory for Innovative System Design recently set up by ST-Microelectronics and the University of Bologna. At European level he is, or has been, a member of the LongTerm Research Advisory Group; of the Joint NSF-CEC Strategy Group, and of the Pathfinder Committee. 

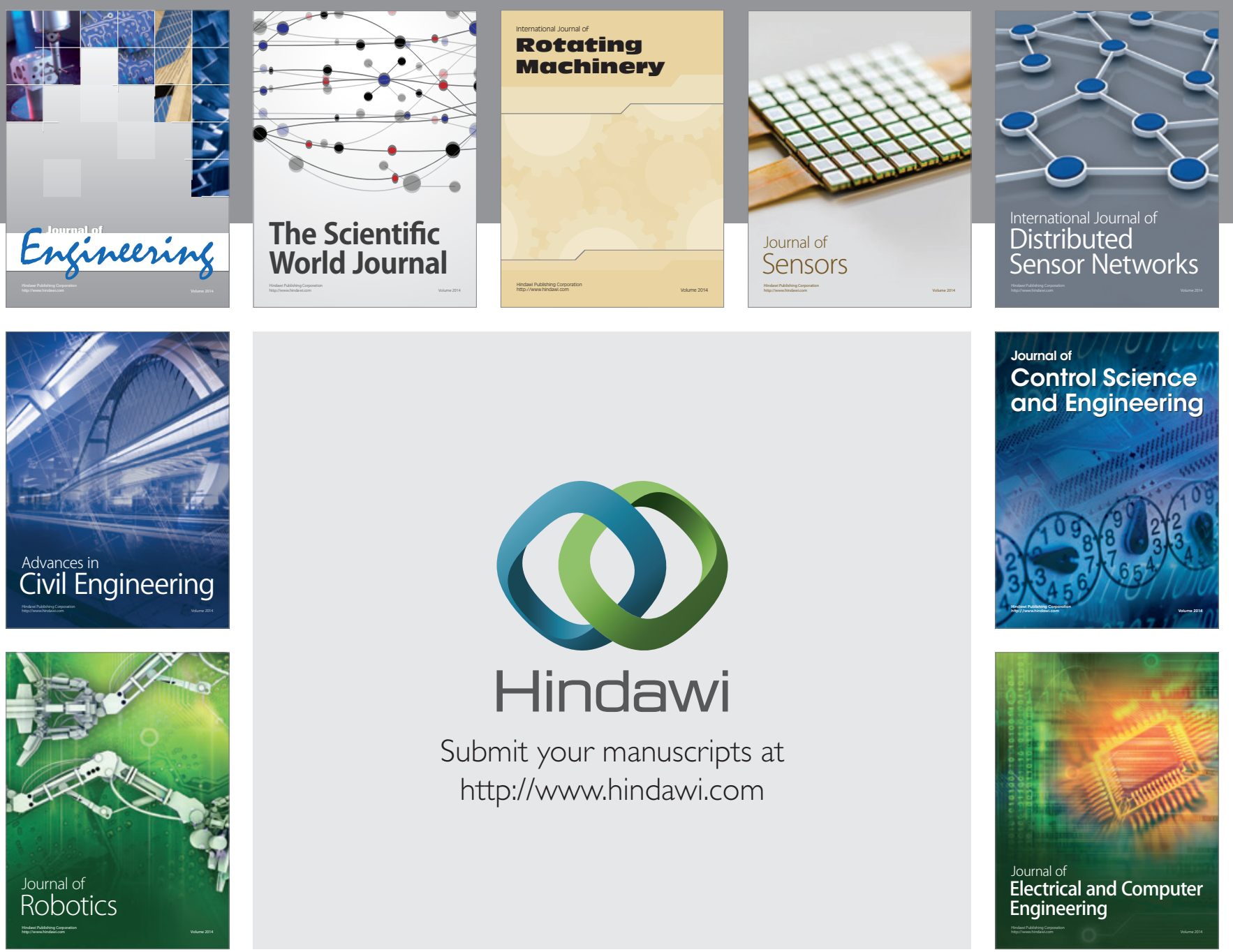

Submit your manuscripts at

http://www.hindawi.com
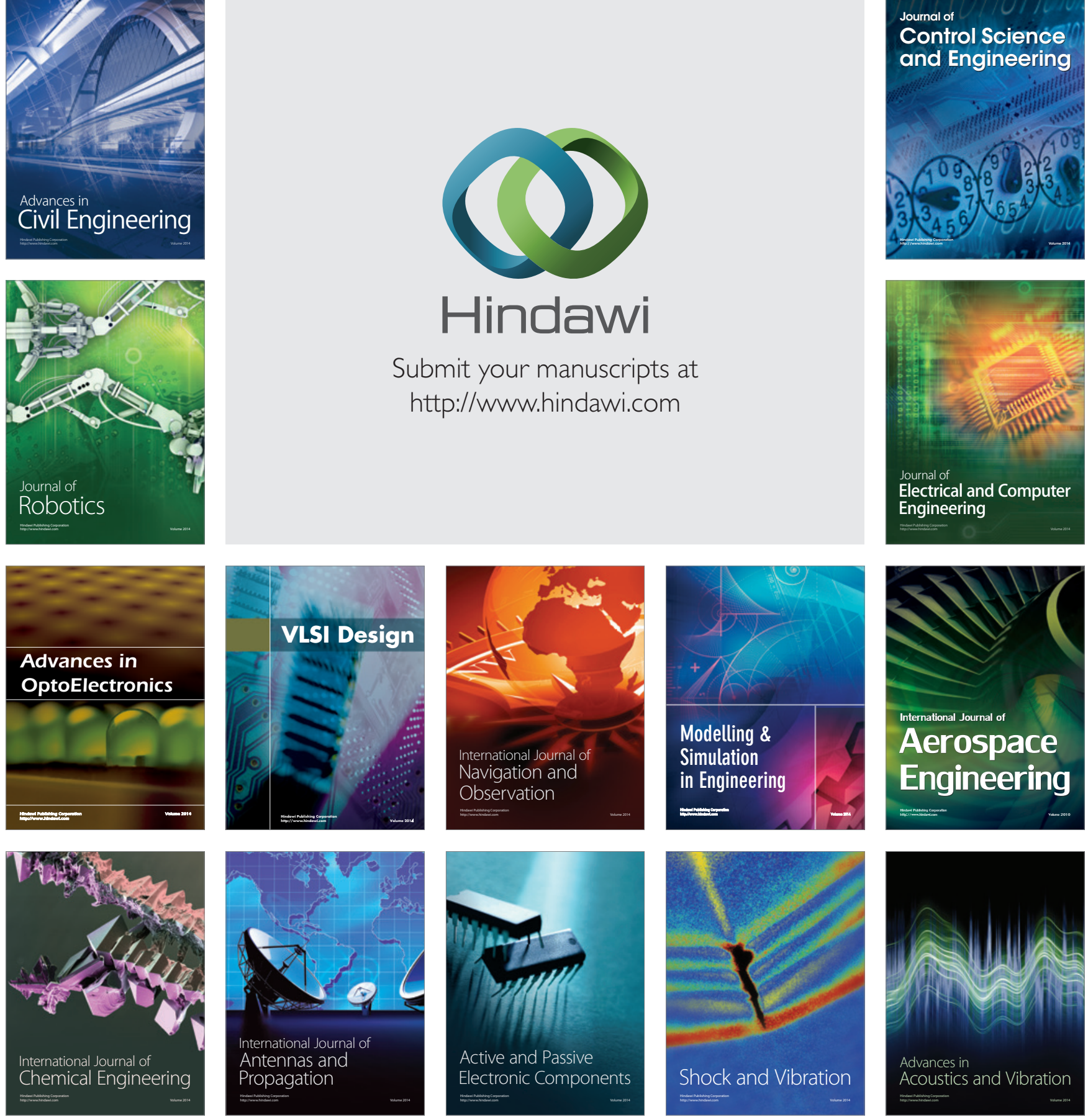